\title{
TAGUNG
}

\section{Zur Rolle Interinstitutioneller Vereinbarungen und des Geschäftsordnungsrechts in der Verfassungsentwicklung der Union}

\author{
Daniela Kietz*
}

Interinstitutionelle Vereinbarungen (IIVs) zwischen den Organen der Europäischen Union sind so alt wie die Union selbst. Dennoch fehlt es bis heute an einem einheitlichen, in der Rechts- und Politikwissenschaft allgemein akzeptierten Verständnis ihrer Rolle und Funktion im Institutionen- und Rechtssystem der Union. Sie sind Teil der rechtlichen Grauzone, zu der auch die Geschäftsordnungspraxis der EU-Organe zählt. Eine gemeinsame Tagung des Arbeitskreises Europäische Integration, der Stiftung Wissenschaft und Politik und des Instituts für Europäische Integrationsforschung Wien versuchte, beide Phänomene und ihr Zusammenspiel interdisziplinär zu beleuchten.

Grundlagen der Interinstitutionellen Vereinbarungen

Um sich diesem sehr spezifischen, komplizierten Thema zu nähern und eine gemeinsame Grundlage für die weiteren Beiträge zu schaffen, wurde zum Auftakt der Tagung versucht, das ,Instrument “ Interinstitutionelle Vereinbarung und seine Funktion im politischen System der Union zunächst einmal aus rechtswissenschaftlicher Sicht zu erfassen und zu bewerten. Bis heute gründen Vereinbarungen zwischen den EU-Organen auf keiner expliziten Rechtsgrundlage in den Verträgen der Union. Ihre rechtliche Zulässigkeit kann nur implizit anhand des in Art. 10 EGV festgelegten Grundsatzes der Gemeinschaftstreue abgeleitet werden. Diese schreibt die Pflicht zu loyaler Zusammenarbeit und ge-

\section{Verfassungsentwicklung zwischen den Europäischen Gipfeln - Chancen und Grenzen des intra- und interinstitutionellen Soft law in der Europäischen Union}

Gemeinsame Tagung des Arbeitskreises Europäische Integration, der Stiftung Wissenschaft und Politik (SWP) und des Instituts für Europäische Integrationsforschung der Österreichischen Akademie der Wissenschaften, Wien

Berlin, 2. Juni 2005

Wissenschaftliche Leitung u. Begrüßung Dr. Andreas MAURER, SWP, Berlin Prof. Dr. Sonja PUNTSCHER RIEKMANN, Österreichische Akademie der Wissenschaften, Wien

Interinstitutionelle Abkommen (IIAs) in der Europäischen Union

50 Jahre IIAs - aus Sicht des Europarechts Prof. Dr. Waldemar HUMMER, Universität Innsbruck

IIAs im Bereich des Budgetverfahrens Isabella EISELT, Dr. Johannes POLLAK, Dr. Peter SLOMINSKI, Österreichische Akademie der Wissenschaften, Wien

IIAs im Legislativprozess der EU

Daniela KIETZ, SWP, Berlin

IIAs in der GASP

Dr. Andreas MAURER

Discussant: Dr. Daniel THYM, WalterHallstein-Institut, Berlin

* Daniela Kietz, Forschungsgruppe EU-Integration, Stiftung Wissenschaft und Politik, Berlin. 
genseitiger Rücksichtnahme zwischen Mitgliedstaaten und der Gemeinschaft vor; durch die Rechtsprechung des EuGH wurde dieses Prinzip allerdings auch auf die Zusammenarbeit der Gemeinschaftsorgane untereinander ausgeweitet. Darauf aufbauend können laut der Erklärung Nummer 3 in der Schlussakte des Vertrags von Nizza EU-Organe, wenn im Rahmen der loyalen Zusammenarbeit notwendig, Interinstitutionelle Vereinbarungen schließen. Die Erklärung unterstreicht jedoch explizit, dass hiermit nur trilaterale Vereinbarungen legitimiert werden und setzt noch zwei weitere rechtliche Schranken: IIVs dürfen die Vertragsbestimmungen weder ändern noch ergänzen. Diese Eingrenzungen sind eine Reaktion auf die Vielzahl der über die Jahre geschlossenen bilateralen Vereinbarungen zwischen Parlament und Kommission, deren Rückwirkungen auf den Rat von diesem als Bedrohung seiner Position im institutionellen Machtdreieck der Union empfunden wurden. Mit der Einigung auf die Erklärung wurde die Hoffnung verbunden, diesen, Wildwuchs' bilateraler Interorganvereinbarungen einzudämmen. Der hier zum Tragen kommende, vom EuGH entwickelte normative Grundsatz des institutionellen Gleichgewichts geht auf das den Gemeinschaftsverträgen zu Grunde liegende Prinzip der begrenzten Einzelermächtigung zurück. Demnach darf erstens die Gemeinschaft nur dort tätig werden, wo sie explizit dazu in den Verträgen durch die Mitgliedstaaten ermächtigt wird (vertikale Kompetenzteilung). Zweitens dürfen die EU-Organe selbst auf keinen Fall - etwa über Interorganvereinbarungen - Kompetenzen untereinander delegieren (horizontale Kompetenzteilung) und dadurch die von den Verträgen etablierte Machtbalance ändern. Dies steht nur den Mitgliedstaaten auf dem Weg der formellen Vertragsänderung zu. Aus rechtlicher Perspektive ist die institutionelle Machtbalance somit statischer Natur. Ob dennoch und wann genau durch IIVs Kompetenzen zwischen den Organen verschoben werden, ist schwierig feststellbar. Aus rechtlicher Perspektive sollten zumindest IIVs, die EUOrganen im Vertrag nicht vorgesehene mate-
Intrainstitutionelle Entwicklungen im Geschäftsordnungsrecht der Organe

Die Reformen innerhalb des EP

Dr. Johann SCHOO, Europäisches Parlament Direktor Juristischer Dienst, Brüssel Geschäftsordnungen und Interinstitutionelle Abkommen als Gratwanderung zwischen praeter und contra Primärrecht

Dr. Moritz RÖTTINGER, Europäische Kommission, Brüssel

Intraorganisationelle Reformen der EuroGruppe und des Wirtschafts- und Finanzausschusses

Dr. des. Daniela SCHWARZER, SWP, Berlin

Chancen und Grenzen der Nutzung Interinstitutioneller Reforminstrumente Die Verfahren und Perspektiven der Finanziellen Vorausschau im Kontext der Verfassungsdebatte

Peter BECKER, SWP, Berlin

Entwicklungen beim Aufbau des Europäischen Auswärtigen Dienstes

Franco ALGIERI, CAP, München

Legitimität und Demokratie durch Interinstitutionelle Abkommen

Prof. Dr. Sonja PUNTSCHER RIEKMANN

rielle Beschlussrechte einräumen und die somit das institutionelle, Gleichgewicht" definitiv verschieben, unzulässig sein. Zulässig hingegen könnten IIVs sein, die einem Organ lediglich Mitwirkungsrechte im Sinne von Informations- und Konsultationsrechten zusprechen.

Die IIVs unterscheiden sich in Bezeichnung, Form, Inhalt und rechtlicher Erfassung. Erst 1988 wurde der einheitliche Begriff ,Interinstitutionelle Vereinbarung' geprägt. Daneben gelten bi- und trilaterale Gemeinsame Erklärungen, Entschließungen, Briefwechsel, Übereinkünfte et cetera als IIVs. Zudem werden IIVs an ganz unterschiedlichen Stellen, teilweise als Anhänge anderer Dokumente veröffentlicht. Inhaltlich wurden sie zunächst im Haushaltsbereich angewendet, weiteten 
sich im Laufe der Zeit aber auf andere Bereiche der interinstitutionellen Kooperation aus wie auf das Rechtsetzungsverfahren und die Komitologie. Diese diversen Erscheinungsformen Interinstitutioneller Vereinbarungen machen eine systematische sozialwissenschaftliche und rechtliche Erfassung und Auswertung der Funktionen und Rechtswirkung von IIVs weit gehend unmöglich.

Allein Einzelfallanalysen sind der Schwachpunkt, wenn es um die Frage geht, ob IIVs unzulässigerweise die Kompetenzverteilung zwischen den Organen ändern. Die Wirkung einer einzelnen IIV mag vernachlässigbar sein, die Masse an Vereinbarungen zusammen kann aber durchaus ein Organ zu Lasten eines anderen stärken. Durch IIVs können Mitwirkungsrechte eines EU-Organs in einzelnen Politikbereichen peu à peu und wenig beachtet gestärkt werden und erst das Gesamtbild zeigt, wie auf diesem informellem Weg des ,intrakonstitutionellen Wandels' das Machtverhältnis zwischen Rat, Kommission und Parlament verschoben wird. Durch den segmentierten Charakter dieser Prozesse und die fehlende Dokumentation kann diese Entwicklung in ihrer Gesamtheit nicht präzise abgeschätzt werden.

Den Abschluss dieser einleitenden rechtlichen Überlegungen bildete die quantitativ untermauerte Feststellung, dass Interinstitutionelle Vereinbarungen überdurchschnittlich häufig das Europäische Parlament begünstigen. Der Löwenanteil an IIVs ist bilateraler Natur, abgeschlossen zwischen Parlament und Kommission beziehungsweise Rat. Das Parlament war zudem überdurchschnittlich oft die treibende Kraft hinter dem Abschluss. Laut Hummer ist den meisten IIVs gemeinsam, dass sie ,vor allem dazu dienen, dem Europäischen Parlament gründungsvertraglich nicht eingeräumte Kompetenzen zur Beteiligung am Willensbildungsprozess- bzw. Rechtsetzungsprozess in den Gemeinschaften im all- gemeinen bzw. an den Tätigkeiten der Organe im speziellen $[\ldots]$ zu gewähren"1. Das Parlament hat im formalen Prozess der Vertragsänderung kein Beschlussrecht und somit auch keine Möglichkeit sich für die Stärkung der eigenen Rolle im Institutionengefüge einzusetzen. Entsprechend liegt der strategische Rückgriff auf informelle IIVs nahe, um seine Mitwirkungsmöglichkeiten graduell zu verbessern.

\section{IIVs als Mittel zur Stärkung des Europäischen}

\section{Parlaments}

In diesem Zusammenhang wurde im Anschluss folgende These auf der Tagung vorgestellt und von Referenten und Teilnehmern diskutiert: durch die sukzessive Ausweitung seiner formalen Legislativ- und Kontrollrechte seit der Einheitlichen Europäischen Akte wurde das formale Verhandlungspotenzial des Parlaments gestärkt. Es nutzt dieses Potenzial als Hebel, um seinen Einfluss auch in Bereichen auszuweiten, in denen ihm seiner eigenen Interpretation nach weitere Mitwirkungsrechte zustehen. Dies geschieht vor allem durch den Abschluss Interinstitutioneller Vereinbarungen mit der Kommission und dem Rat, die dem Parlament auf informellen Weg vertraglich nicht vorgesehene Rechte zusprechen. Neben der Befugnis zur Ablehnung des Haushaltsplanes ist es vor allem die Einführung des Mitentscheidungsverfahrens und die dadurch eröffnete Möglichkeit der Verzögerung und Verwerfung von Gesetzesvorlagen, die das Verhandlungs- beziehungsweise Drohpotenzial des Parlaments stärkten und die interinstitutionelle Machtverteilung insofern veränderten, als dass sie das Europäische Parlament als gleichberechtigten Akteur neben Kommission und Rat etablierten. Als gleichberechtigter Ko-Gesetzgeber standen dem Parlament seiner eigenen Auffassung zufolge aber auch Mitwirkungsrechte in den ebenfalls zum Gesetzgebungsprozess gehörenden Bereichen wie bei der Delegation und

1 Waldemar Hummer: Interinstitutionelle Vereinbarungen und ,institutionelles Gleichgewicht“, in: Waldemar Hummer (Hrsg.): Paradigmenwechsel im Europarecht zur Jahrtausendwende. Ansichten österreichischer Integrationsexperten zu aktuellen Problemlagen. Forschung und Lehre in Österreich, Wien 2004, S. 116. 
Kontrolle von Durchführungsmaßnahmen (Komitologie) zu. Es war für das Parlament unannehmbar geworden, dass der Rat als nur ein Teil der Legislative die ausschließliche Kontrolle über und ein Rückholrecht für die Durchführungsmaßnahmen für gemeinsam mit dem Parlament beschlossene Rechtsakte besaß. Das Europäische Parlament forderte, dass Rechtsakte, die es mit dem Rat gemeinsam delegierte, auch nur gemeinsam kontrolliert und im Notfall zurückgeholt werden sollten. In der Folge schloss das Parlament mit der Kommission beziehungsweise mit Kommission und Rat eine Reihe von IIVs ab - die wohl bekannteste ist der Modus Vivendi aus dem Jahr 1994 -, die seine Informationsrechte hinsichtlich den in Komitologieausschüssen behandelten Entwürfen für Durchführungsmaßnahmen ausweiteten. 1999 erneuerte der Rat die formale, aus dem Jahre 1987 stammende rechtliche Grundlage für die Komitologie, den Beschluss über die Festlegung der Modalitäten für die Ausübung der der Kommission übertragenen Durchführungsbefugnisse. Auf dem Modus Vivendi aufbauend, überträgt der neue Beschluss dem Parlament bei der Durchführung von Rechtsakten, die im Mitentscheidungsverfahrens erlassen werden, ein Mitspracherecht.

Eng verbunden mit beziehungsweise Teil dieser ,Kompetenzerweiterungspolitik ' des Parlaments ist nämlich die parlamentarische $\mathrm{Ge}$ schäftsordnungspraxis. Diese bildete einen weiteren Schwerpunkt der Tagung. Wie IIVs dient die Geschäftsordnung dem Parlament als ein strategisches Instrument zur eigenen Machtstärkung. Sie sieht Rechte und Verpflichtungen der Kommission und des Rates gegenüber dem Parlament vor, die weit über die Regelungen der Verträge hinausgehen. Ein Großteil der praktizierten Ernennungsprozedur der Kommission, wie etwa die individuellen Anhörungen der Kommissare in den parlamentarischen Ausschüssen, sind primärrechtlich nicht vorgesehen. Ein Teil dieser einseitig erklärten Vorschriften sind durch IIVs abgesichert, die häufig der Geschäftsordnung angehängt werden. Mittlerweile sind die
Beziehungen zwischen Kommission und Parlament auf Grundlage der Verträge und ohne das Zusammenspiel von einzelnen Artikeln der Geschäftsordnung und Interinstitutioneller Rahmenvereinbarung zwischen beiden Organen nicht mehr nachvollziehbar. In den letzten Jahren zeichnet sich zudem ein Trend $\mathrm{ab}$, nach dem IIVs durch die Geschäftsordnung implementiert werden.

\section{IIVs in wichtigen Politikfeldern der EU}

Weitere Tagungsbeiträge beleuchteten die Rolle Interinstitutioneller Vereinbarungen in wichtigen Politikbereichen der Union. Es findet sich beispielsweise in der Innen- und Justizpolitik und der Gemeinsamen Außen- und Sicherheitspolitik (GASP) eine Reihe an IIVs. Deren Hauptgegenstand ist wiederum die Ausweitung der Einflussmöglichkeiten des Parlaments in diesen Feldern. Generell fußt in der GASP ein beträchtlicher Teil der institutionellen Entwicklungen nicht auf formalen Vertragsermächtigungen. Neueres Beispiel ist der sich im Vorgriff auf das In-Kraft-Treten des Verfassungsvertrags bereits im Aufbau befindliche Europäische Auswärtige Dienst. In den gültigen Verträgen findet sich keine explizite Ermächtigungsgrundlage für seine Implementierung. Falls die Ratifizierung des Verfassungsvertrags scheitern sollte, wird die Möglichkeit der Umsetzung des Dienstes mittels Abschluss einer IIV als durchaus realistisch eingeschätzt. Ein weiteres Organ, das ganz ohne Rechtsgrundlage und sogar ohne Geschäftsordnung arbeitet, ist die so genannte Euro-Gruppe zur wirtschaftspolitischen Koordination der zwölf Mitglieder der Euro-Zone. Sie verfügt weder über Mandat, offiziellen Namen, Sekretariat oder ähnliches, sondern stützt sich auf die Strukturen des ECOFINRates. In der Annahme des baldigen Beitritts aller Mitgliedstaaten zur Euro-Zone sollte ihre Informalität eigentlich die weitere Einheit und damit die Vermeidung der Schaffung eines formellen Gremiums neben dem Rat gewährleisten. Im Lauf der letzten Jahre wurde jedoch ein Großteil der Entscheidungen in die Gruppe ausgelagert beziehungsweise dort 
vorentschieden. Im Verfassungsvertrag beugen sich die Mitgliedstaaten dieser Realität, erkennen die Funktion der Gruppe an und formalisieren ihre mittlerweile institutionalisierten Treffen.

Gleich zwei Beiträge befassten sich mit dem wohl bekanntesten Fall Interinstitutioneller Vereinbarungen, den Abkommen im Rahmen der mehrjährigen Haushaltsplanung. Anlässlich der Haushaltsreform im Jahr 1988 kamen Parlament, Rat und Kommission in einer IIV überein, sich jeweils im Voraus über die Haushaltsprioritäten eines festgelegten, mehrjährigen Zeitraums zu einigen. Diese so genannten Finanziellen Vorausschauen sind den seither in regelmäßigen Abständen geschlossenen IIVs zur „Haushaltsdisziplin und die Verbesserung des Haushaltsverfahrens" (1988, 1993, 1999) beigestellt. In diesen Vereinbarungen etablieren die Organe konkrete Konfliktlösungsmechanismen für ihre Zusammenarbeit bei der Erstellung des Haushaltsplans. Die mehrjährige Finanzplanung mit dem Instrument der IIV wurde mit dem Ziel eingeführt, den bereits seit den 1970er Jahren schwelenden Machtkampf zwischen Rat und Parlament im Bereich der Haushaltspolitik zu beenden. Ein seit der Beteiligung des Parlaments an der Verabschiedung des jährlichen Haushalts bis heute nicht gelöster Streitpunkt ist die Trennung der Ausgaben in obligatorische und nicht obligatorische. Das Parlament hat lediglich ein Mitspracherecht in der Festsetzung der Ausgaben der zweiten Kategorie. Entsprechend führte die Kategorisierung der Ausgabenbereiche und die Allokation der Mittel in den 1980er Jahren immer wieder zu scharfen Konflikten zwischen dem Rat und dem - nicht zuletzt durch seine Direktwahl seit 1979 und durch die Einheitliche Europäische Akte in seinen Rechten gestärkten - Parlament. Während dieses versuchte, über die Zuteilung beziehungsweise die Einfügung völlig neuer Haushaltslinien vermehrt auf den Inhalt der Politiken Einfluss zu nehmen, versuchte der Rat, über die Festschreibung von Höchstbeträgen in den von ihm verabschiedeten Rechtsakten die Haushaltsrechte des Par- laments zu umschiffen. Nach mehreren aufeinander folgenden Haushaltskrisen und Verzögerungen bei der Annahme des jährlichen Haushaltsplans, Klageserien vor dem EuGH bis hin zu Ablehnung des Planes durch das Parlament (1980, 1985) einigte man sich 1988 auf die Haushaltsreform. Insgesamt haben IIVs den Ablauf des Haushaltsverfahrens deutlich verbessert. Durch die Selbstbindung aller drei Organe an ein förmliches Verfahren der Zusammenarbeit etablierten die IIVs einen verbindlichen Rahmen für die Diskussionen und die Lösung der interinstitutionellen Konflikte. Auch wenn bis heute immer wieder Querelen bei der Verabschiedung des Haushalts auftreten, wird das Konfliktlösungspotenzial der IIVs in diesem Bereich als hoch eingeschätzt.

\section{IIVs als Instrumente zur Lösung interinstitutio- neller Konflikte}

Dieser Blick über die Politikfelder ließ die Tagungsteilnehmer zu dem Schluss kommen, dass IIVs hauptsächlich Instrumente zur Lösung interinstitutioneller Konflikte darstellen. Sie wirken vor allem dort, wo in den Verträgen die Kompetenzen der Organe beziehungsweise die Kooperation zwischen ihnen vage formuliert sind. Dort jedoch, wo die Vertragsvorschriften vage sind oder sich widersprechen, ist Freiraum für Interpretation. Wie ein roter Faden zog sich das Argument durch die Tagung, dass das Parlament versucht, seine Interpretation der ihm vertraglich zustehenden Kompetenzen durch IIVs durchzusetzen. Mit anderen Worten, es versucht, strategisch seine Kompetenzen über IIVs auszuweiten. Entgegen der juristischen Sichtweise, kommt aus politikwissenschaftlicher Sicht der, weichen' Mitwirkung am Entscheidungsprozess (Informations- und Konsultationsrechte) wie durch die Einspeisung von Positionen über Stellungnahmen oder Initiativberichten eine große Bedeutung zu. Um das Parlament zu stärken, müssen IIVs ihm nicht notwendigerweise, harte' Entscheidungsbefugnisse übertragen. Ein weiteres eher politik- als rechtswissenschaftlich erfassbares Phänomen 
ermöglicht die Kompetenzausweitung über IIVs: Informelle IIVs können den Weg für spätere formale Änderungen des Sekundärund sogar des Primärrechts ebnen und sind in diesem Sinne Träger eines vor allem von der historisch-institutionalistisch arbeitenden Politikwissenschaft betonten inkrementellen institutionellen Wandels. Sie schaffen in der informellen Arena irreversible Fakten durch die Einführung und Institutionalisierung politischer Prozesse, hinter denen spätere Primäroder Sekundärrechtsgebung nicht zurückbleibt, sondern aufbaut. Über diese, die formalen Vertragrevisionen vorstrukturierenden Wandlungsprozesse zwischen den eigentlichen Regierungskonferenzen kann das Europäische Parlament - ohne in die formalen Prozesse der Vertragsänderungen einbezogen zu sein - seine Position im Institutionengefüge stärken und sich als informeller AgendaSetter betätigen. Die Entwicklung der parlamentarischen Mitwirkungsrechte in der Komitologie bis hin zur Verabschiedung des Komitologiebeschlusses aus dem Jahr 1999 oder die Kodifizierung der Euro-Gruppe im Verfassungsvertrag sind beispielhaft für solche Pfadabhängigkeiten.

\section{Schlussfolgerungen}

Die Tagung zeigte, dass die Untersuchung von IIVs vor allem eine Auseinandersetzung mit dem andauernden Parlamentarisierungsund Demokratisierungsprozess der Union ist. Die Frage nach der Rolle solcher Vereinba- rungen in der Union musste also zum $\mathrm{Ab}$ schluss der Tagung von den Referenten auch aus demokratietheoretischer Sicht beantwortet werden. Stärken IIVs durch ihre Tendenz zur Aufwertung des Parlaments das demokratische Fundament der Union? Ausgehend von einem repräsentativ-demokratischen Verständnis von Demokratie ist eine Stärkung des Parlaments positiv zu beurteilen. Folgt man jedoch der Argumentation, dass demokratische (Input-)Legitimität an das Vorhandensein eines Europäischen Demos gebunden ist, was bisher für die Union verneint werden muss, führt eine Stärkung des Parlaments nicht unmittelbar zu einer Demokratisierung des Systems. Aus dieser Perspektive bleiben weiterhin die nationalen Parlamente und über sie die Regierungen im Rat sowie im Falle von Referenden die Bürger Hauptlegitimationsträger der Entscheidungen. Der informelle, schrittweise Weg der Kompetenzübertragung an das Europäische Parlament mittels IIVs, der sich zu einem großen Teil dem Einfluss dieser Legitimationsträger entzieht, muss dann kritisch betrachtet werden. Zudem tragen IIVs durch ihre informelle Natur und Diversität nicht zur Erhöhung der Transparenz der politischen Entscheidungsprozesse in der Union bei. Bei Einbezug output-orientierter Legitimationsargumente schneiden IIVs wiederum besser ab, da sie konfliktentschärfend wirken und damit die Effizienz der Entscheidungsfindung erheblich erhöhen. 